\title{
The distribution and impact of common copy-number variation in the genome of the domesticated apple, Malus x domestica Borkh
}

\author{
James Boocock ${ }^{1,2^{*}}$, David Chagné 2,3 , Tony R. Merriman ${ }^{1,2}$ and Michael A. Black ${ }^{1,2^{*}}$
}

\begin{abstract}
Background: Copy number variation (CNV) is a common feature of eukaryotic genomes, and a growing body of evidence suggests that genes affected by CNV are enriched in processes that are associated with environmental responses. Here we use next generation sequence (NGS) data to detect copy-number variable regions (CNVRs) within the Malus $\mathrm{x}$ domestica genome, as well as to examine their distribution and impact.

Methods: CNVRs were detected using NGS data derived from 30 accessions of $\mathbf{M} \times \mathbf{x}$ domestica analyzed using the read-depth method, as implemented in the CNVrd2 software. To improve the reliability of our results, we developed a quality control and analysis procedure that involved checking for organelle DNA, not repeat masking, and the determination of CNVR identity using a permutation testing procedure.

Results: Overall, we identified 876 CNVRs, which spanned $3.5 \%$ of the apple genome. To verify that detected CNVRs were not artifacts, we analyzed the B- allele-frequencies (BAF) within a single nucleotide polymorphism (SNP) array dataset derived from a screening of 185 individual apple accessions and found the CNVRs were enriched for SNPs having aberrant BAFs ( $P<1 \mathrm{e}-13$, Fisher's Exact test). Putative CNVRs overlapped 845 gene models and were enriched for resistance $(R)$ gene models $(P<1 \mathrm{e}-22$, Fisher's exact test). Of note was a cluster of resistance gene models on chromosome 2 near a region containing multiple major gene loci conferring resistance to apple scab.

Conclusion: We present the first analysis and catalogue of CNVRs in the M. $x$ domestica genome. The enrichment of the CNVRs with $\mathrm{R}$ gene models and their overlap with gene loci of agricultural significance draw attention to a form of unexplored genetic variation in apple. This research will underpin further investigation of the role that CNV plays within the apple genome.
\end{abstract}

\section{Background}

The availability of genome sequence data from individuals within a species now enables the investigation of a range of inherited genetic variations at a high resolution. Traditionally, genomic analysis of DNA variants has focused on the identification of single nucleotide polymorphisms (SNPs), and small insertions and deletions (INDELS). However in recent years, other forms of genomic variation have also begun to receive attention. One such form is copy number variation $(\mathrm{CNV})$, defined as a deletion, duplication or insertion of DNA sequence fragments longer than 50 base pairs in length [1].

\footnotetext{
*Correspondence: james.boocock@otago.ac.nz; mik.black@otago.ac.nz 'Department of Biochemistry, University of Otago, Dunedin, New Zealand Full list of author information is available at the end of the article
}

Studies of CNV in eukaryotic organisms, such as dog [2], barley [3], and human [4], have revealed that 4 to $15 \%$ of a eukaryotic genome is comprised of regions which exhibit variation in copy number between individuals. CNVs have the potential to exert influence on genes by altering both their expression and structure. For example, in humans the common Sotos syndrome generally occurs when a deletion of one copy of the plasma coagulation 12 (FXII) gene exposes a deficiency in the remaining copy [5]. CNV can also contribute to the phenotypic diversity of domesticated animals: in cattle, a partial deletion of the ED1 gene causes anhidrotic ectodermal dysplasia [6]; and in pigs, white coat color is caused by a duplication involving the KIT gene $[7,8]$. CNV distribution is not random across genomes. As an example, segmental duplications (SDs),
C Biomed Central

(c) 2015 Boocock et al. Open Access This article is distributed under the terms of the Creative Commons Attribution 4.0 International License (http://creativecommons.org/licenses/by/4.0/), which permits unrestricted use, distribution, and reproduction in any medium, provided you give appropriate credit to the original author(s) and the source, provide a link to the Creative Commons license, and indicate if changes were made. The Creative Commons Public Domain Dedication waiver (http://creativecommons.org/publicdomain/zero/1.0/) applies to the data made available in this article, unless otherwise stated. 
which are sections of DNA with near-identical sequence, are considered hotspots for CNV formation [9].

$\mathrm{CNV}$ is a common feature of plant genomes, and recent work has highlighted its functional relevance. In barley, genes affected by $\mathrm{CNV}$ were enriched for potential functions in disease resistance [3], a finding that is consistent with the results of CNV studies in soybean [10] and maize [11]. In wheat, CNVs in Vrn-A1 and Ppd-B1 have been shown to influence flowering time [12], and a CNV at the $R h t-D 1 b$ locus is associated with dwarfism [13]. In soybean, increased copy-number of an allele of the Rhg1 gene is responsible for nematode resistance [14], while in barley, boron tolerance is associated with a CNV at the Bot1 locus [15]. Based on these reports, there is evidence that CNVs are frequently associated with the biotic and abiotic stress response in annual crop species. However, no analysis of CNVs in perennial tree species has been performed to date. We hypothesize that CNVs are particularly relevant as a source of genetic variability that can be rapidly utilized for adaptation to stress in long-lived plants, including horticultural and forest tree species.

The domesticated apple (Malus x domestica Borkh.) first appeared in the Near East around 4,000 years ago. In 2010, a high-quality draft genome of 'Golden Delicious' was released [16]. Pairwise comparison of the assembled genome revealed that a whole genome duplication (WGD) had recently $<50$ million years ago occurred in the Pyrinae (which includes fruit species such as apple, pear and quince), leading to an almost doubling of chromosomal number. However, non-global duplication and deletion events, which are localized to individual loci or regions and can thus be considered to represent CNV, have not previously been described in the apple genome. Apple researchers have used Next-Generation Sequencing (NGS) technologies to detect SNPs across the genome, enabling the development of apple SNP arrays used for genomic selection in apple breeding programs and for fine trait mapping [17-19]. NGS-based re-sequencing in the apple genome has indicated that domesticated apple is significantly heterozygous, with 4.8 SNPs per kb, and a recent study has revealed that multiple introgression events with wild apple species such as Malus sylvestris and M. sieversii have shaped the genomes of modern domesticated cultivars [20].

Hybridization-based methods, such as array comparative genomic hybridization (aCGH), were the first highthroughput approaches used to identify CNVs at the genome-wide scale [21]. More recently however, NGS technologies combined with new analytical approaches, such as read-depth, paired-end, and split-reads analysis, have become popular [22]. Analysis of read-depth is an effective method for CNV detection, relying on detection of changes in the depth of coverage across the genome as being indicative of changes in the underlying copy-number [23]. The aim of our study was to detect CNV regions (CNVRs), in the apple genome using lowcoverage $(1 \mathrm{x}$ to $5 \mathrm{x})$ NGS re-sequencing data from 30 apple varieties grown or used for cultivar breeding worldwide.

\section{Methods}

Plant material and next-generation sequencing

A low-coverage NGS dataset for 30 domesticated apple (M. $\mathrm{x}$ domestica) accessions was developed using Illumina GAII technology, with one lane per individual as described in [19]. These varieties represent founders, intermediate ancestors, or important breeding parents used extensively in apple breeding programs worldwide. The complete set maximized coverage of the genetic background of the world's domesticated apple. Individuals in the set were Malus x domestica 'Braeburn', 'CrimsonCrisp,' 'Delicious', (both the original form and the derived mutant 'Red Delicious'), 'Duchess of Oldenburg,' F2268292-2, 'Geneva,' 'Golden Delicious,' 'Granny Smith, 'Haralson, 'Honeycrisp', 'Idared,' 'James Grieve,' 'Jonathan,' 'McIntosh,' Ralls Janet,' 'Red Dougherty, 'Rome Beauty, 'Splendour, 'Big Red,' 'Papa Noel', 'Merton Russet,' 'Wilmont Russet,' 'David' o.p., 'Malling 9', and four advanced selections. The raw sequencing data for many of the accessions is publicly available, and for the other accessions data can be made available on request (Additional file 1).

\section{Sequence alignment and CNVR identification}

The reads from the 30 Malus $\mathrm{x}$ domestica samples were aligned to the pseudohaplotype assembly of 'Golden Delicious' [16] using the Burrow's wheeler aligner (BWA v 0.7.5a) maximal exact matches (MEM) command [24, 25]. To reduce the impact of highly abundant organelle DNA on the read depth analysis, a LAST (v 6.21) [26] database was created using apple mitochondrial DNA [27] and apple chloroplast DNA [28]. Sections of the assembly with a highly confident match (score $>=40$ ) were considered to be derived from organelles. Additionally, the locations of repeat sequences, such as retrotransposons, were obtained [29] and reads that overlapped repeat sequences, mitochondrial, or chloroplast locations by at least one base pair were removed using bedtools (v2.19.1) [30]. As duplications can occur during the PCR amplification process and create spurious spikes in read-depth signal, these were identified and removed using Picard's ( $v$ 1.124) MarkDuplicates functionality [31].

The binary alignment format (BAM) files used for CNV analysis were filtered based on mapping quality (reads with MAPQ $<20$ were discarded), and processed using CNVrd2 [32] (v1.4.0), where the read-depth was counted in 3000-bp non-overlapping windows. Following this, windows that covered a segment of the assembly with greater than $50 \%$ Ns were removed. The GC-content of a genomic region 
influences the read-depth when using Illumina sequencing [33]. This presents a challenge for read-depth analysis and was minimized using the GC-content adjustment method that is implemented in $\mathrm{CNVrd2}$. The resulting GC-adjusted read count data were then standardized within and between samples using CNVrd2. As a result of standardizing across multiple samples, the influence of mappability bias, which is present at some complex regions, was minimized. Coverage statistics were generated using the $\mathrm{R}$ programming language (v3.1.1) [34] with figures created in $\mathrm{R}$ using the ggplot2 (v1.0.0) [35] and gplots (v2.16) [36] packages. CNVrd2 uses the DNACopy package [37] from the Bioconductor project [38], which employs a binary segmentation algorithm to segment the normalized read-counts. This procedure results in a segmentation score for each window in each sample. These scores represent significant changes in read-depth, with numbers greater than zero indicating a change to increased read-depth, and numbers less than zero indicating a change to decreased read-depth. These changes in read-depth are indicative of changes in the underlying copy-number within a sample. Although these raw segmentation scores can be processed to obtain integer copy-numbers, for this work we chose to analyze the raw segmentation scores, focusing on detecting regions that varied significantly in segmentation score between the apple accessions. Spearman's correlation was used to investigate the relationship between read-depth and number of segmented regions. A visualization of the distribution of CNVRs throughout the apple was created using Circos (v0.67-4) [39]. In addition to detecting CNVRs with CNVRd2, an independent analysis was performed for comparison using the filtered BAM files with the R package cn.MOPS [40].

To identify CNVRs, a trimmed standard deviation (removing the min. and max. values) was calculated to compare the segmentation scores in each sample for each window. This trimmed standard deviation was used to remove outliers from the calculations. Permutation testing (10,000 permutations per chromosome) was used to determine CNVR significance. This involved shuffling the segmented regions in each sample, recalculating trimmed standard deviations, and calculating an empirical false discovery rate (FDR) [41]. The FDR was calculated at different thresholds, with the genome-wide FDR calculated as a mean of each chromosomal value weighted by chromosome length. A genome-wide trimmed standard deviation threshold of 0.25 was used to determine whether a window was within a CNVR, and neighboring windows above this threshold were merged to form the CNVRs.

\section{SNP Array support for candidate CNVRs}

A SNP dataset was used for validation of CNVRs which contained information on the scoring of 10,685 SNP polymorphic markers from the apple Illumina Infinium $20 \mathrm{k} \mathrm{SNP}$ array [18] screened over 185 apple accessions from the Plant \& Food Research germplasm collection that have a similar genetic background to the varieties used for read-depth analysis. As the $20 \mathrm{k} \mathrm{SNP}$ array data comes from an independent experiment that utilized microarray technology, it presented us with an opportunity to strengthen the evidence that the CNVRs detected with read-depth from sequencing data were reliable. SNP genotyping assumes diploid copy number, an assumption that is violated when a SNP falls within CNVRs, as genotypes such as $\mathrm{AAB}$, carrying an extra copy of the "A" allele, and $A \varnothing$, missing a copy of the "B" allele, may be present [42]. The $B$ allele frequency (BAF), which is the proportion of signal explained by the $\mathrm{B}$ allele, has an expected value for heterozygotes of 0.5 , and for homozygotes of 0 or 1 . Odd numbers of alleles at a locus, such as $\mathrm{AAB}$, may give values falling outside these values. The $\mathrm{B}$ allele frequency (BAF) was extracted for each SNP data point using the Illumina GenomeStudio software. CNVRs were tested for enrichment of SNP markers with a B allele frequency (BAF) between 0.05 and 0.35 , or 0.65 and 0.95 , in at least $10 \%$ (19) of samples. Fisher's exact test [43] was used to check whether the array design was biased against SNPs being located in CNVRs.

\section{Gene model annotation and functional analysis}

The consensus gene models were obtained from the Genome Database for Rosaceae [44]. A gene model was considered to overlap a CNVR if more than $70 \%$ of its bases were within the boundaries of a CNVR. Gene Ontology analysis [45] was performed using the Fisher's exact test. The resulting $P$-values were adjusted using the FDR-controlling approach of Benjamimi and Hochberg [41]. A separate enrichment test was performed using predicted resistance gene models obtained from the supplementary information of the publication describing the apple genome [16]. The repeat sequences were classified further by BLAST searching [46] against the RepBase19.12 [47] database. The flanking regions of CNVRs were determined by creating a list of positions $10 \mathrm{~kb}$ either side of each CNVR, and merging the overlapping regions. The number of elements for each class that entirely overlapped or did not overlap was calculated, followed by enrichment testing using a Fisher's exact test. Genic regions were determined by creating a list of positions $10 \mathrm{~kb}$ either side of each gene model. The average GC content of the flanking regions of both the CNVRs and the genic regions was calculated on a per chromosome basis.

\section{Results}

Data summary and CNVR identification

Before quality control $(\mathrm{QC})$, the average sequencing coverage for each variety varied between $3.51 \times$ and $13.60 \times$. After 
QC, which included removing reads overlapping the organelle and repeated regions, the average sequencing coverage for each variety was reduced to between $0.95 \times$ and $4.96 \times$ (Additional file 1), indicating that between 63 and $73 \%$ of the reads mapped to the excluded regions.

The read-depth CNV detection method is based on an assumption that the number of reads originating from a region of a genome after removing technical bias is indicative of the copy number for that region. Reads were counted in 3-kb non-overlapping windows. Prior to normalization the median read-depth for all windows was 38. Following GC-content adjustment the median read-depth became 16.8, this highlights the impact that GC content can have on read-depth. After normalization, these windowed read counts were segmented into regions with similar signal values. Investigation of the relationship between coverage and the number of segmented regions revealed a positive correlation (Spearman's correlation of 0.656) between coverage and the number of regions detected, which is an indication that CNVs are more likely to be detected in samples of higher coverage (Fig. 1). We used a linear model to further quantify this relationship, regressing the number of segmented regions against sequencing coverage. The beta estimate from this model was 82.58 $(P<1 \mathrm{e}-5, T$-test $)$, meaning that for every unit increase in average coverage; the model estimated that the average number of segmented regions increased by 82.58. Although integer copy number assignment for an individual sample can be performed using the read-depth method, given the low-coverage sequencing data used in our study and the incomplete apple genome assembly, we chose instead to focus on CNVRs that displayed significant variation in segmentation scores and not to attempt integer copy number assignment. These significantly variable CNVRs were determined by summarizing each window using a trimmed standard deviation (removing the minimum and maximum), followed by a permutation testing procedure to calculate the threshold used to identify potential CNVRs: any window with a trimmed standard deviation above this threshold was considered to lie within a CNVR. A threshold of 0.25 gave an acceptable FDR of $11 \%$, and was used in downstream analyses (Fig. 2).

The 876 CNVRs detected using the above threshold spanned a total of $14.4 \mathrm{Mb}$ or $3.5 \%$ of the 'Golden Delicious' v1.0p pseudohaplotype assembly (Additional file 2). They ranged in size from $3 \mathrm{~kb}$ to $99 \mathrm{~kb}$, with an average length of $16.4 \mathrm{~kb}$, and a median length of $12 \mathrm{~kb}$ (Fig. 3). CNVRs appeared to be non-randomly distributed throughout the genome (Fig. 4). The percentage of an individual chromosome in CNVRs varied between $1.5 \%$ for chromosome 16 and $5.7 \%$ for chromosome 10 (Additional file 3).

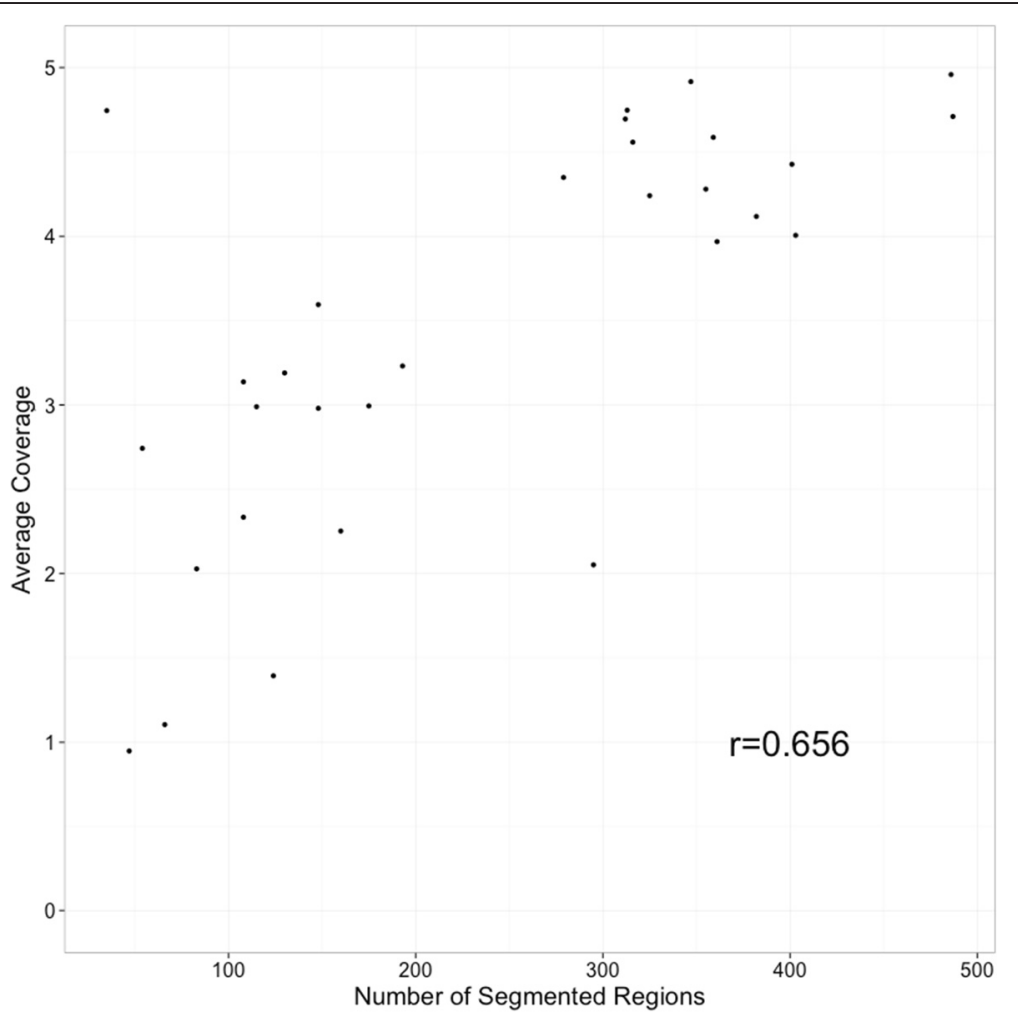

Fig. 1 Relationship between number of segmented regions detected in each apple accession and the apple genome coverage. Average coverage per apple accession versus total number of segmented regions, determined using CNVrd2 

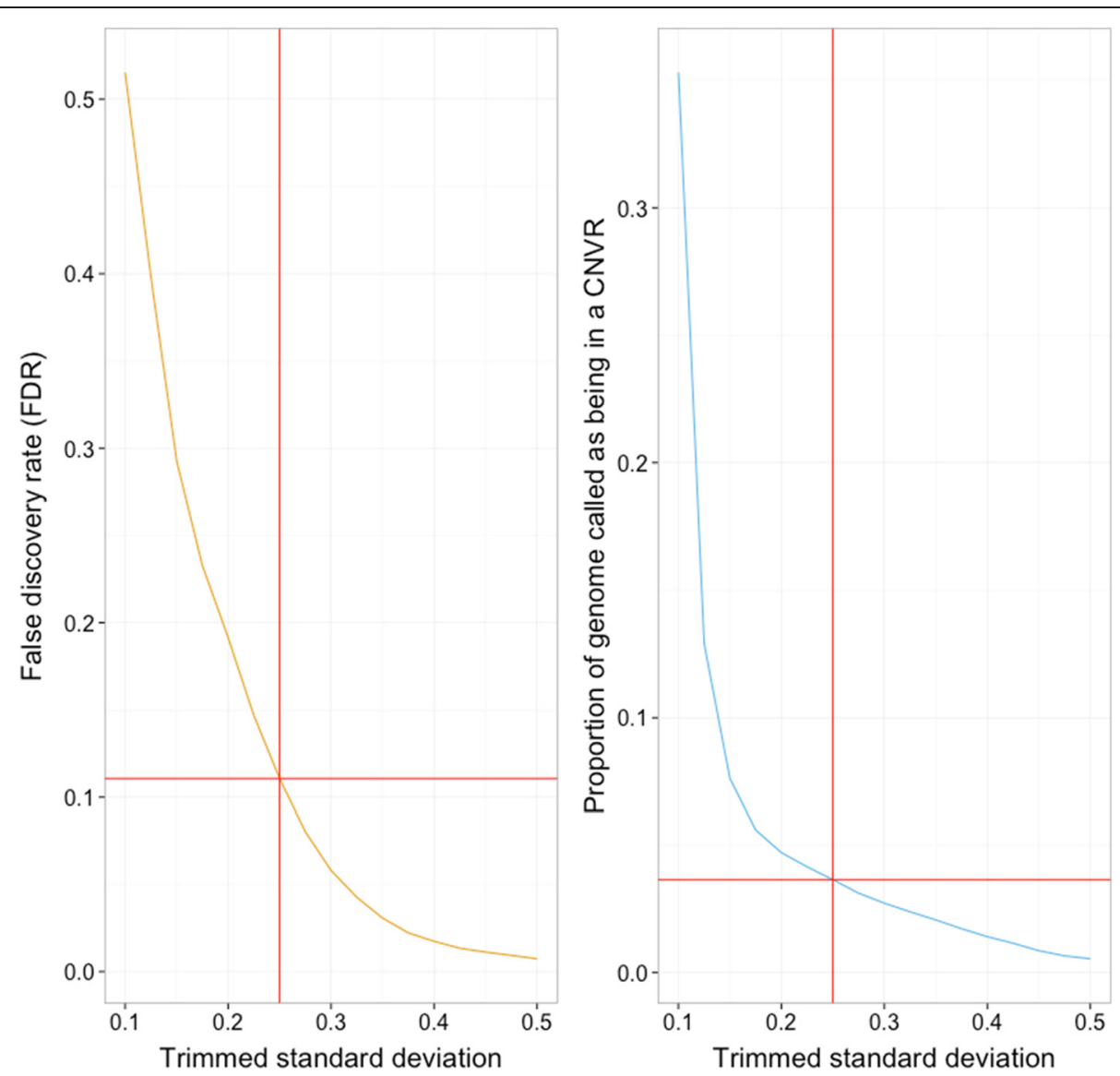

Fig. 2 Relationship between the false discovery rate (FDR) estimated using permutation analysis, and the proportion of the genome comprising copy-number variable regions (CNVRs) in the apple genome, as a function of significance threshold. The red lines intersect at a threshold of 0.25 , which was the threshold used for downstream CNV analysis using re-sequencing data of 30 apple accessions

When the removal of regions split a large CNVR, it was considered as two smaller CNVRs. In addition to the readdepth analysis performed using CNVRd2, CNVRs were detected with the $\mathrm{R}$ package cn.Mops (data not shown). These CNVRs based on cn.Mops analysis spanned a total of $41.8 \mathrm{Mb}$ or $10 \%$ of the apple genome. In total, $59.6 \%$ of the exact bases in the CNVRs were detected both by CNVRd2 and cn.Mops. If one relaxes the criteria and counts the entire $\mathrm{CNV}$ length reported from CNVRd2 when an overlap is observed, then $87.5 \%$ of the CNVR bases were recovered.

\section{Repeat analysis and GC content}

Repeated sequences were investigated for their relationship with CNVRs. A list of 10-kb regions flanking CNVRs was developed, with overlapping sections merged. The repeated elements that are the most numerous in the apple genome (Copia, Gypsy, hAT, Cassandra, and LINE) [16] were tested for enrichment within the flanking regions. A significant depletion was observed for Gypsy elements $(P=0.007$, Fisher's exact test) and a significant enrichment for Copia elements $(P=0.006$, Fisher's exact test) (Table 1$)$. No significant enrichments or depletions $(P>0.05$, Fisher's exact test) were observed for $h A T$, Cassandra, and LINE elements, and no overall enrichment $(P>0.05$, Fisher's exact test) was detected for repeated elements. The genome-wide GC content of CNVRs was nominally $(P=0.03, T$-test $)$ higher (average $37.9 \%$ ) than that of the pseudohaplotype assembly (37.8\%). In contrast, the genome-wide average for genic GC content was nominally $(P=0.02, T$-test) lower than that of CNVRs (average $37.6 \%$ ) (Additional file 3).

\section{Independent experimental support for candidate CNVRs using a SNP array dataset}

Although low density SNP microarrays do not enable copy-number detection directly, because of their limited probe density, they do present a method of independently validating CNVRs detected by read-depth analysis. We accomplished this by extracting the BAFs from an apple Illumina Infinium 20-k SNP array dataset that contained the genotyping information for 185 accessions. After the removal of SNPs that overlapped the windows which were previously removed from the analysis because they contained Ns, repeats, or organelle DNA, 10,685 SNPs 


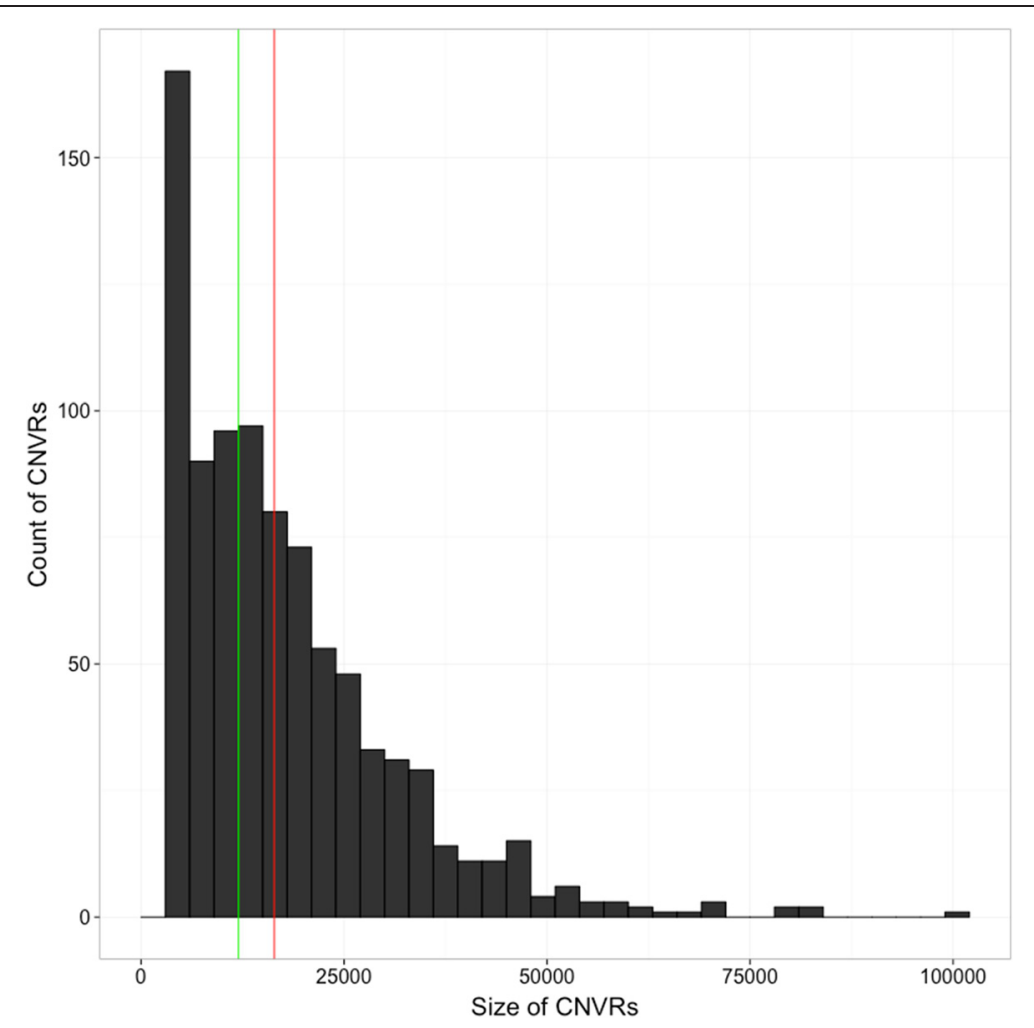

Fig. 3 Distribution of length and frequency of copy-number variable regions (CNVRs) in the apple genome. Size (in base pairs) versus number of CNVRs. The green and red lines represent the median and mean CNVR size, respectively

remained. BAF thresholds of $0.05-0.35$ and $0.65-0.95$ were considered aberrant (Fig. 5) and when 19 (10\%) of samples fell within these ranges, the SNPs were considered as having an aberrant BAF pattern indicative of CNV. CNVRs contained 49 of the 1845 (2.6\%) SNPs with aberrant BAFs, and 115 of the 8840 (1.3\%) normal (disomic) SNPs, representing an approximately two-fold enrichment of SNPs with aberrant BAFs, within CNVRs $(P<0.0001$, Fisher's Exact test). Interestingly, $98.5 \%$ of the SNPs represented on the apple Illumina Infinium $20 \mathrm{k}$ SNP array were located in nonCNV regions, indicating a significant depletion of SNPs in CNVRs $(P<1 \mathrm{e}-13$, Fisher's Exact test) and suggests that the SNP array design was biased away from CNVRs.

\section{Functional analysis of CNVRs}

Of the consensus gene models from the Genome Database for Rosaceae, 29,015 were located in genomic regions that were analyzed. A total of $845(2.9 \%)$ of these gene models exhibited a minimum of $70 \%$ of their base pairs overlapping putative CNVRs (Additional file 4). Significant depletion of gene models within CNVRs was observed $(P<1 \mathrm{e}-6$, Fisher's Exact test), with the proportion of the genome assembly spanned by CNVRs (3.5\%) being greater than the proportion containing gene models. Over half (470) the CNVRs did not contain a gene model, with the remaining 406 regions (46.3\%) containing at least one gene model. Functional enrichment analysis of CNVRs revealed 20 GO terms overrepresented after FDR $p$-value adjustment (Table 2). These terms included "apoptotic process", "innate immune response" and "defense response", for which a significant proportion of annotations were found to originate from resistance ( $R$ ) gene models. This class of genes are proteins containing nucleotide-binding sites (NBS or NBC-ARC domains) and C-terminal leucine-rich repeats (LRR), and are key components of the immune response in plants $[48,49]$. To investigate the relationship between $\mathrm{R}$ gene models and CNVRs directly, we obtained a list of 992 resistance $(R)$ gene models from the apple genome publication [16]. Of the $992 \mathrm{R}$ gene models, 268 were located in the GD pseudohaplotype assembly we used for CNV detection, and 47 (16.3\%) of these R gene models overlapped CNVRs (Additional file 5). Furthermore, R gene models were enriched greater than five-fold within CNVRs (5.5 \% of total gene models) compared with outside these regions, where they comprised $0.8 \%$ of total gene models $(P<1 \mathrm{e}-22$, Fisher's exact test) (Table 3$)$. This significant enrichment of $\mathrm{R}$ genes is consistent with results from the cn.MOPS analysis $(P<1 \mathrm{e}-47$ for cn.MOPS results only; $P<1 \mathrm{e}-19$ for the intersection between cn.MOPS and CNVrd2). Ten of these enriched 


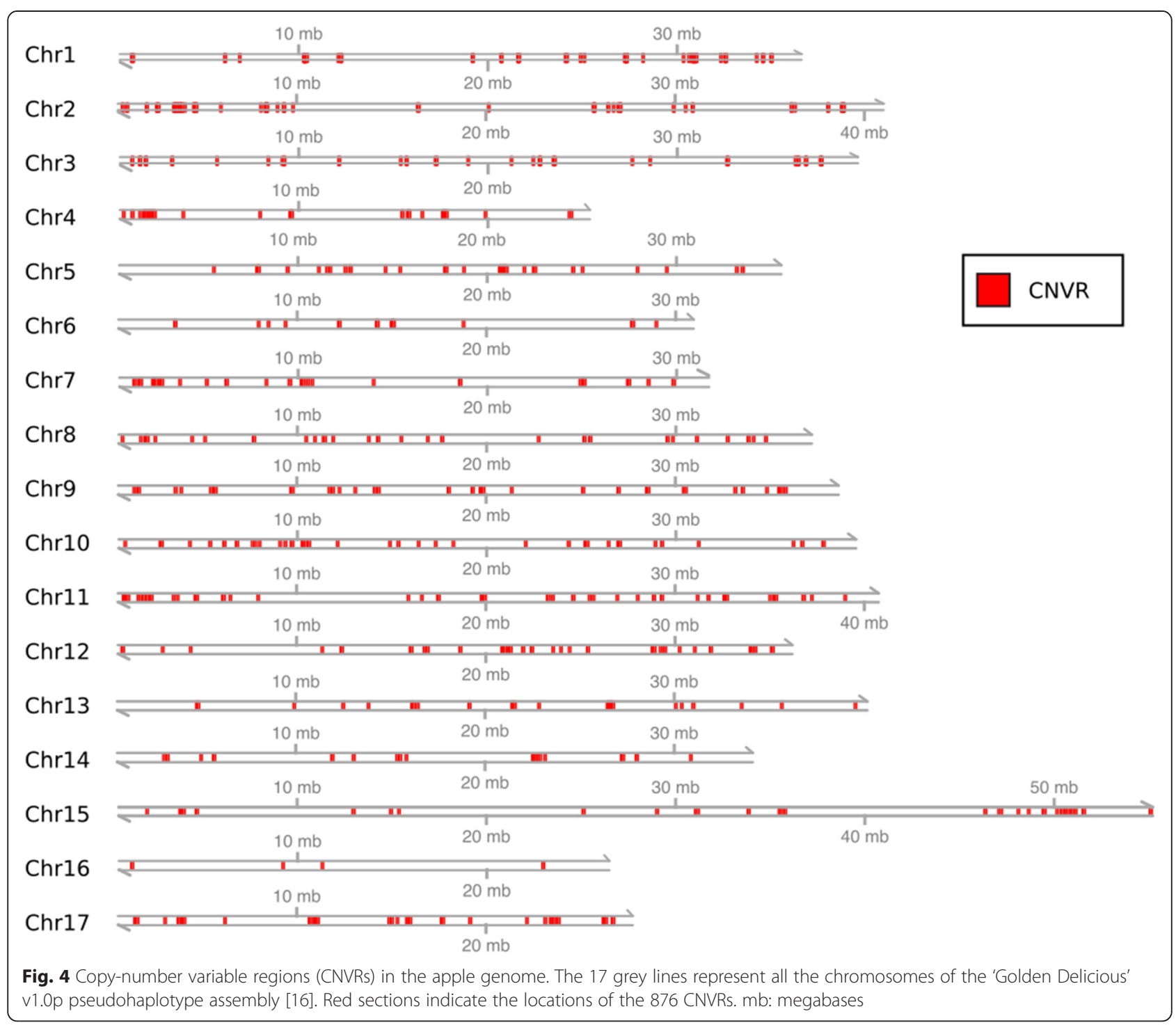

GO terms were not attributable to $\mathrm{R}$ gene models, such as "ion transport", "chloride transport", and "voltagegated chloride channel activity".

\section{Discussion}

Characterization of the apple genome has focused primarily to date on whole genome duplication, the detection of SNPs, the prediction of genes and the characterization of gene families, as well as generation of an inventory of repeated elements. Structural variations, such as copynumber, have not been studied in detail, in apple or indeed in other fruit tree species. In short-lived crop species, such as maize and rice, copy-number variation has been investigated using NGS and microarray technologies, and a growing consensus has emerged as to the agricultural relevance of $\mathrm{CNV}$ [52]. In particular, genes located in CNVRs have been observed to be enriched for stress-related response genes, including the canonical R-genes, which contain a leucine rich-repeat (LRR) domain. This highlights the importance of $\mathrm{CNV}$ in relation to agricultural crop genetics, and the need to characterize this type of variation in horticultural species. In this study, we used NGS data from 30 apple accessions to identify 876 copy-number variable regions (CNVRs). The CNVRs within the apple genome overlap 845 gene models and are enriched for R gene models. A significant enrichment of SNPs with aberrant BAFs was observed within CNVRs by using a SNP derived from a screening of 185 individual apple accessions with similar genetic background to those of the 30 varieties used for read-depth analysis. This strengthens the evidence that the regions we detected represent true CNVRs.

Although CNV had not been investigated previously in the apple genome, there has been some evidence that $\mathrm{CNV}$ might play an important biological role here. A study investigating QTLs associated with bud phenology in apple identified a candidate E3 ubiquitin protein encoding gene 
Table 1 Number of repetitive elements contained in the $10 \mathrm{~kb}$ of sequence up- and downstream of the copy-number variable regions (CNVRs) in the apple genome

\begin{tabular}{|c|c|c|c|c|c|}
\hline \multicolumn{3}{|c|}{ CNVR flanking Regions } & \multicolumn{3}{|c|}{ Non-CNV flanking regions } \\
\hline Repeat type & Observed & Expected & Observed & Expected & $P$-value \\
\hline Gypsy & 1917 & 2087.5 & 85,346 & 85175.5 & 0.007 \\
\hline Copia & 815 & 707.7 & 28,767 & 28874.3 & 0.006 \\
\hline Cassandra & 245 & 225.2 & 9169 & 9188.8 & 0.375 \\
\hline LINE/RTE & 264 & 251.5 & 10,250 & 10262.5 & 0.624 \\
\hline hAT & 402 & 350.6 & 14,253 & 14304.4 & 0.065 \\
\hline Total & 3643 & 3622.4 & 147,785 & 147805.6 & 0.812 \\
\hline
\end{tabular}

present as a tandem array of 14 copies in 'Golden Delicious', making it a putative $\mathrm{CNV}$ [50]. More recently, a study that was performed to design SNP markers for eight major disease resistance loci, encountered two problems that can be explained by the occurrence of CNV in these regions [51]. Firstly, for the Rvi2, Rvi4 and Rvi11 loci conferring resistance to apple scab (Venturia inaequalis), the presence of paralogs made it difficult to design primers for the SNPs linked to the respective loci, which amplified alleles only at the specific region of interest, without coamplifying the paralogous sequences. Paralogs located in tandem would be considered a segmental duplication, which are hotspots for CNVs [9]. Secondly, SNPs linked to $\mathrm{Pl2}$, a major locus conferring resistance to powdery mildew (Posdosphaera leucotricha), failed to amplify when resistance was not observed. This would be expected if resistance were conferred by the insertion of a segment of DNA, which contains the SNPs that failed to amplify, and is absent from susceptible individuals [51].

\section{Data QC and analysis approach}

Because of apple's high heterozygosity, its genome was not assembled as a single reference sequence but rather into four haplotypes, or individual sequences. The primary pseudohaplotype assembly (Malus x domestica Whole Genome v1.0p) [53], which was used in this analysis, represents the shortest path through the sequence scaffolds. This reference was used to facilitate short-read mapping and read-depth analysis, using a workflow analogous to studies in diploid mammals, for which many bioinformatic tools have been developed [22]. Repeated elements, which are estimated to cover $42.4 \%$ of the apple genome [16], create a problem for read-depth analysis, as reads which map to repeat regions of the reference create noise in the read-depth signal. This interferes with data normalization

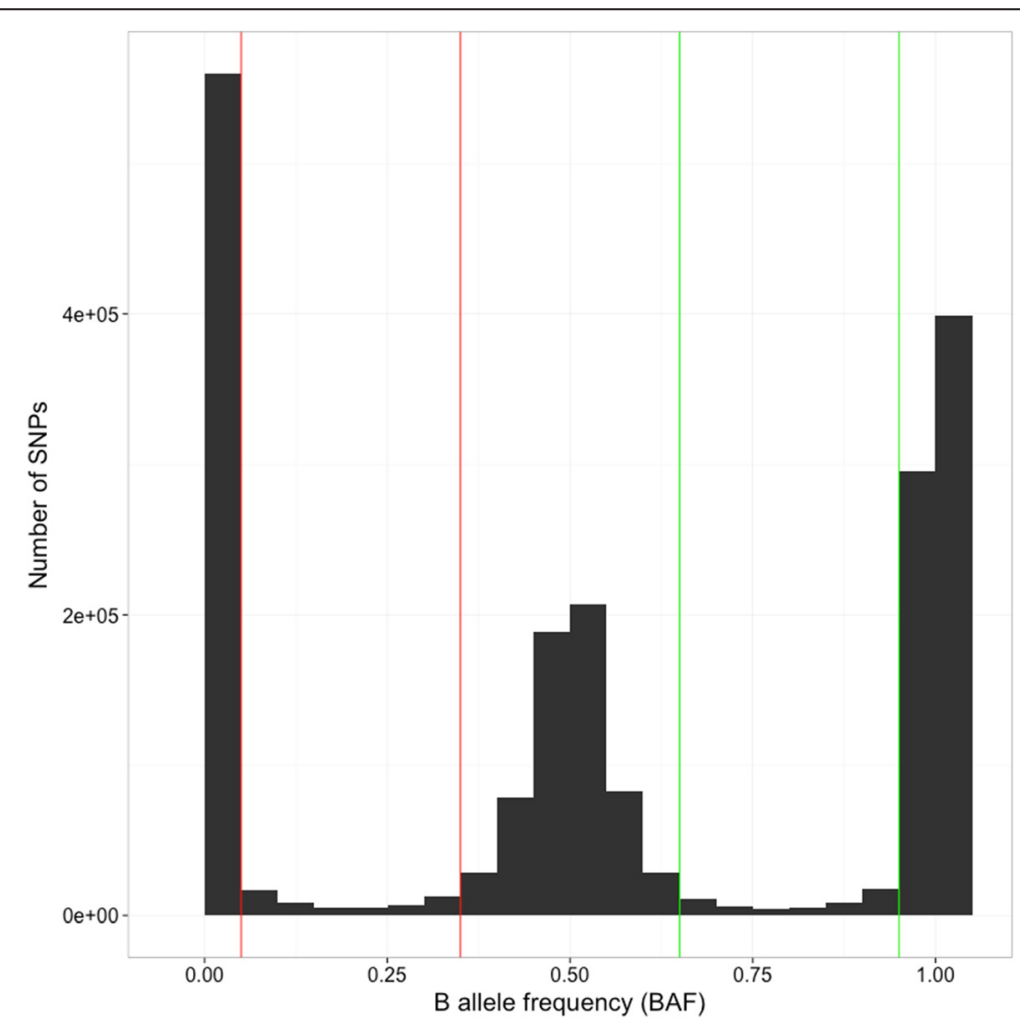

Fig. 5 Distribution of B allele frequencies for the $20 \mathrm{k} \mathrm{SNP}$ array screened on 185 apple accessions. The count of all B allele frequencies (BAFs) is displayed in windows of size 0.05 from a dataset containing 185 apple accessions genotyped on the apple Infinium $20 \mathrm{k}$ SNP array [18]. The ranges $0.05-0.35$ and $0.65-0.95$, shown by red and green lines respectively, indicate the ranges where an individual BAF was considered aberrant 
Table 2 Significant Gene Ontology terms ( $P<0.05$ after False-discovery rate correction) for gene models located within copy-number variable regions (CNVRs) in the apple genome

\begin{tabular}{|c|c|c|}
\hline Geno Ontology ID & Description & $P$ value \\
\hline GO:0006915 & apoptotic process & $4.23 \mathrm{E}-48$ \\
\hline GO:0031224 & intrinsic component of membrane & $4.09 \mathrm{E}-43$ \\
\hline GO:0004888 & transmembrane signaling receptor activity & 1.16E-42 \\
\hline GO:0045087 & innate immune response & $2.75 \mathrm{E}-42$ \\
\hline GO:0005515 & protein binding & 1.03E-32 \\
\hline GO:0007165 & signal transduction & 8.93E-31 \\
\hline GO:0005524 & ATP binding & $9.26 \mathrm{E}-22$ \\
\hline GO:0006952 & defense response & 2.40E-21 \\
\hline GO:0005216 & ion channel activity & 0.006 \\
\hline GO:0006508 & proteolysis & 0.009 \\
\hline GO:0004674 & protein serine/threonine kinase activity & 0.011 \\
\hline GO:0006468 & protein phosphorylation & 0.011 \\
\hline GO:0043086 & negative regulation of catalytic activity & 0.011 \\
\hline GO:0005516 & calmodulin binding & 0.012 \\
\hline GO:0005247 & voltage-gated chloride channel activity & 0.026 \\
\hline GO:0042802 & identical protein binding & 0.028 \\
\hline GO:0004497 & monooxygenase activity & 0.042 \\
\hline GO:0006821 & chloride transport & 0.042 \\
\hline GO:0030552 & CAMP binding & 0.045 \\
\hline GO:0016710 & trans-cinnamate 4-monooxygenase activity & 0.049 \\
\hline
\end{tabular}

and subsequently the downstream segmentation procedure. Many CNV studies attempt to correct for this effect by masking repeat regions before alignment (i.e., changing the position in the reference to an 'N') $[54,55]$. However, as the quality score of reads is determined by the uniqueness of the mapping position, masking may lead to reads that originate from the repeated sequences mapping spuriously to other locations, with potentially high quality scores. In our analysis, reads were first mapped, and only then were repeated elements removed. This represents an alternative and potentially improved approach to overcoming the problem of repeated sequences. The presence of chloroplast and mitochondrial DNA within the reference genome creates a similar issue, as the large number of these organelles in each cell would lead to large numbers of reads mapping to these regions, potentially interfering with the read-depth approach. In the analysis presented here, reads mapping to organelle-derived regions were removed before CNV segmentation.

Low-coverage NGS re-sequencing data were used to identify the CNVRs that exhibited significant variation in copy-number within a set of apple accessions with genetic links to international breeding programs. The CNVRs identified were found to vary greatly in copy-number among the accessions, and represent candidate gene loci that may be involved in control of variability in traits, such as pest and disease resistance. The number of samples used (30 apple accessions) is higher than employed in some recent read-depth studies in domesticated organisms [e.g., the number of samples was five and 16 in $\mathrm{CNV}$ studies of cows [55] and pigs, respectively [54]]; however, is smaller than studies in maize [56] and soybean [10], which involved 278 and 302 samples, respectively. The average coverage for sequencing of our samples ranged from $0.95 \times$ to $4.96 \times$, which is relatively low and might have introduced noise into the read-depth signal. Our finding that a strong correlation was observed in our dataset between sequencing coverage and the number of detected CNVs suggested that the read-depth method is more efficient at detecting copy-number changes in higher-depth samples, and as low-coverage data do not enable reliable calling of exact copy number at specific loci for individuals, we opted to analyze the variation among segmentation scores across the 30 varieties to provide an inventory of CNVRs in the apple genome. A trimmed standard deviation was chosen (removal of the min. and max. values), to reduce the impact of outliers potentially driven by noise, and a permutation test was used to assess the likelihood of finding such a variable region by chance. This allowed us to determine a conservative threshold for CNVR identity, with what we considered an acceptable FDR of $11 \%$ [41]. 
Table 3 Resistance (R) gene models located within copy-number variable regions (CNVRs) in the apple genome

\begin{tabular}{|c|c|c|c|c|}
\hline MPIDs & Chromosome & Start (bp) & End (bp) & Width (bp) \\
\hline MDP0000816743 & chr1 & 24886957 & 24890824 & 3867 \\
\hline MDP0000144874 & chr1 & 30626140 & 30628752 & 2612 \\
\hline MDP0000634037 & chr1 & 30801610 & 30806248 & 4638 \\
\hline MDP0000257201 & chr1 & 30817742 & 30823513 & 5771 \\
\hline MDP0000282740 & chr1 & 30901618 & 30906893 & 5275 \\
\hline MDP0000149577 & chr1 & 31011253 & 31015489 & 4236 \\
\hline MDP0000685087 & chr2 & 2694102 & 2698622 & 4520 \\
\hline MDP0000483490 & chr2 & 2705247 & 2706068 & 821 \\
\hline MDP0000577795 & chr2 & 3531631 & 3532194 & 563 \\
\hline MDP0000478441 & chr2 & 3606656 & 3610584 & 3928 \\
\hline MDP0000522693 & chr2 & 3615408 & 3616082 & 674 \\
\hline MDP0000563856 & chr2 & 3870168 & 3872449 & 2281 \\
\hline MDP0000466397 & chr2 & 3940831 & 3943960 & 3129 \\
\hline MDP0000458576 & chr2 & 3967880 & 3972931 & 5051 \\
\hline MDP0000746482 & chr2 & 3985869 & 3990107 & 4238 \\
\hline MDP0000460947 & chr2 & 4615319 & 4619759 & 4440 \\
\hline MDP0000512349 & chr2 & 4701149 & 4701745 & 596 \\
\hline MDP0000573272 & chr2 & 4731508 & 4735373 & 3865 \\
\hline MDP0000206582 & chr2 & 9335438 & 9338647 & 3209 \\
\hline MDP0000301892 & chr2 & 9357524 & 9362451 & 4927 \\
\hline MDP0000640508 & chr2 & 38880821 & 38885458 & 4637 \\
\hline MDP0000261720 & chr2 & 38910867 & 38912498 & 1631 \\
\hline MDP0000303694 & chr3 & 22748766 & 22753650 & 4884 \\
\hline MDP0000173893 & chr4 & 15497478 & 15501779 & 4301 \\
\hline MDP0000399716 & chr6 & 15009829 & 15020552 & 10723 \\
\hline MDP0000148432 & chr6 & 15038138 & 15040970 & 2832 \\
\hline MDP0000257606 & chr6 & 15042847 & 15050886 & 8039 \\
\hline MDP0000166759 & chr7 & 1337480 & 1340290 & 2810 \\
\hline MDP0000196621 & chr7 & 1370821 & 1373441 & 2620 \\
\hline MDP0000259862 & chr7 & 1591348 & 1594205 & 2857 \\
\hline MDP0000493661 & chr7 & 2442347 & 2446032 & 3685 \\
\hline MDP0000321189 & chr7 & 2458873 & 2466023 & 7150 \\
\hline MDP0000685425 & chr8 & 11405302 & 11410962 & 5660 \\
\hline MDP0000586712 & chr9 & 1526915 & 1531337 & 4422 \\
\hline MDP0000125338 & chr9 & 19770884 & 19773602 & 2718 \\
\hline MDP0000472864 & chr10 & 892484 & 896484 & 4000 \\
\hline MDP0000264448 & chr10 & 10432552 & 10452135 & 19583 \\
\hline MDP0000193087 & chr11 & 1928588 & 1932682 & 4094 \\
\hline MDP0000732188 & chr11 & 2110384 & 2116572 & 6188 \\
\hline MDP0000212932 & chr11 & 3506106 & 3509841 & 3735 \\
\hline MDP0000230932 & chr11 & 3712888 & 3720930 & 8042 \\
\hline MDP0000171723 & chr13 & 21556571 & 21559228 & 2657 \\
\hline MDP0000168665 & chr14 & 13071029 & 13075189 & 4160 \\
\hline MDP0000272830 & chr15 & 15393697 & 15404409 & 10712 \\
\hline
\end{tabular}


Table 3 Resistance (R) gene models located within copy-number variable regions (CNVRs) in the apple genome (Continued)

\begin{tabular}{lllll}
\hline MDP0000680657 & chr15 & 48113339 & 48116942 & 3603 \\
MDP0000312626 & chr17 & 1585061 & 1588664 & 3603 \\
MDP0000291677 & chr17 & 17704304 & 17710114 & 5810 \\
\hline
\end{tabular}

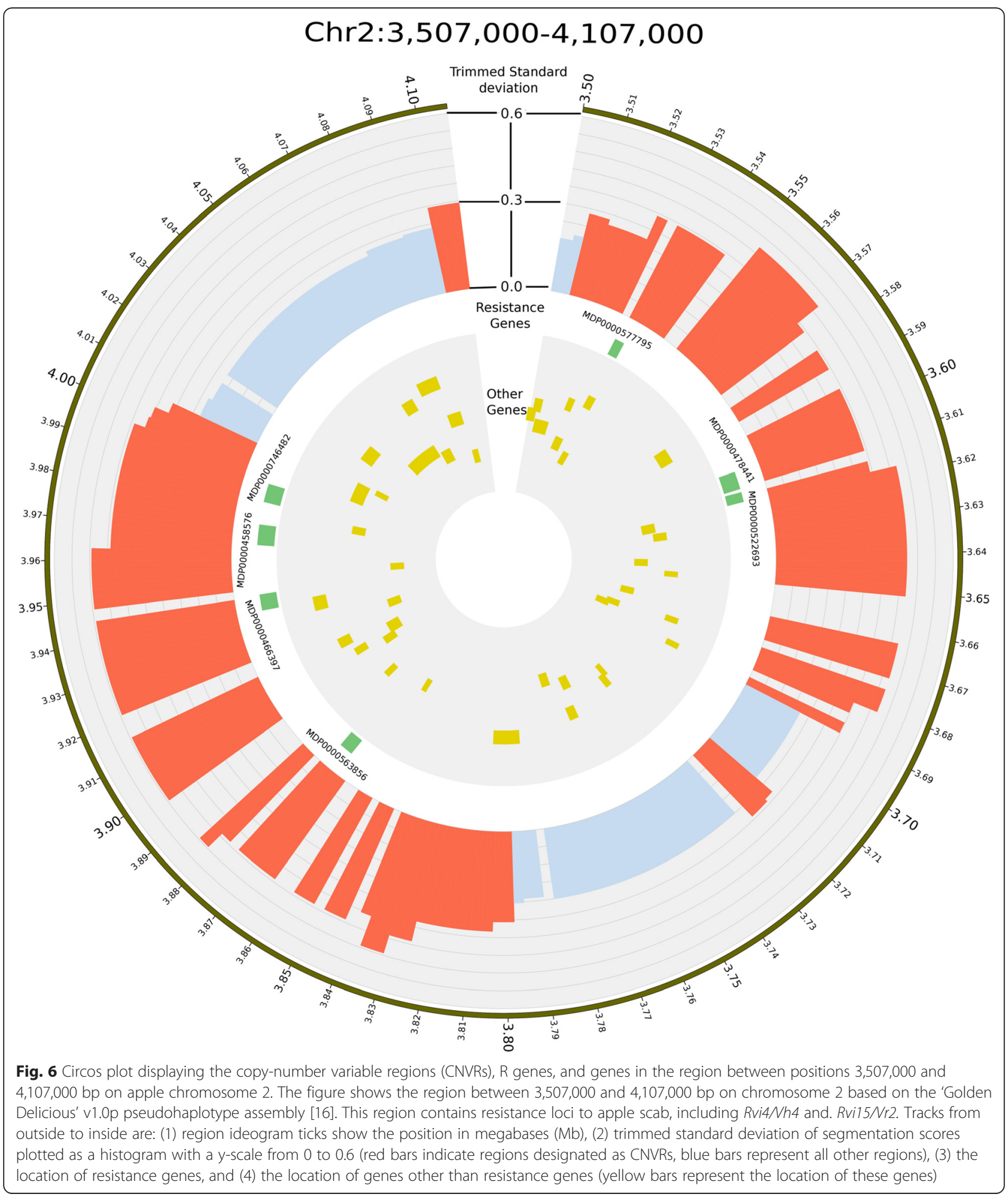




\section{Patterns of CNVRs in the apple genome}

The read-depth analysis identified $876 \mathrm{CNVRs}$ in the apple genome, ranging in length between $3 \mathrm{~kb}$ and $99 \mathrm{~kb}$, with an average of $18 \mathrm{~kb}$. It should be noted that because the CNVRs separated by windows that were removed were not merged, the number of detected CNVRs is likely to be an overestimate, as a number of CNVRs interleaved with removed regions may actually comprise a single large CNVR. Our results suggest that $3.5 \%(14.4 \mathrm{Mb})$ of the analyzed assembly lies within a CNVR. This percentage is lower than found in previous CNV studies in plants; for example, the CNV percentages found in maize [56] and barley [3] were 10 and $14.9 \%$, respectively. This discrepancy might be explained by the lower degree of polymorphism observed among the apple accessions employed in our study compared with that in maize lines, whereby apple has one SNP every 288 bp on average [19], versus every $60 \mathrm{bp}$ in maize [57]. However, it is possible that we might have underestimated the total contribution of CNVs to the apple genome because of the low-coverage sequencing data, strict quality control, and our analysis approach, which focused on detecting only CNVRs that exhibited high variation among the samples.

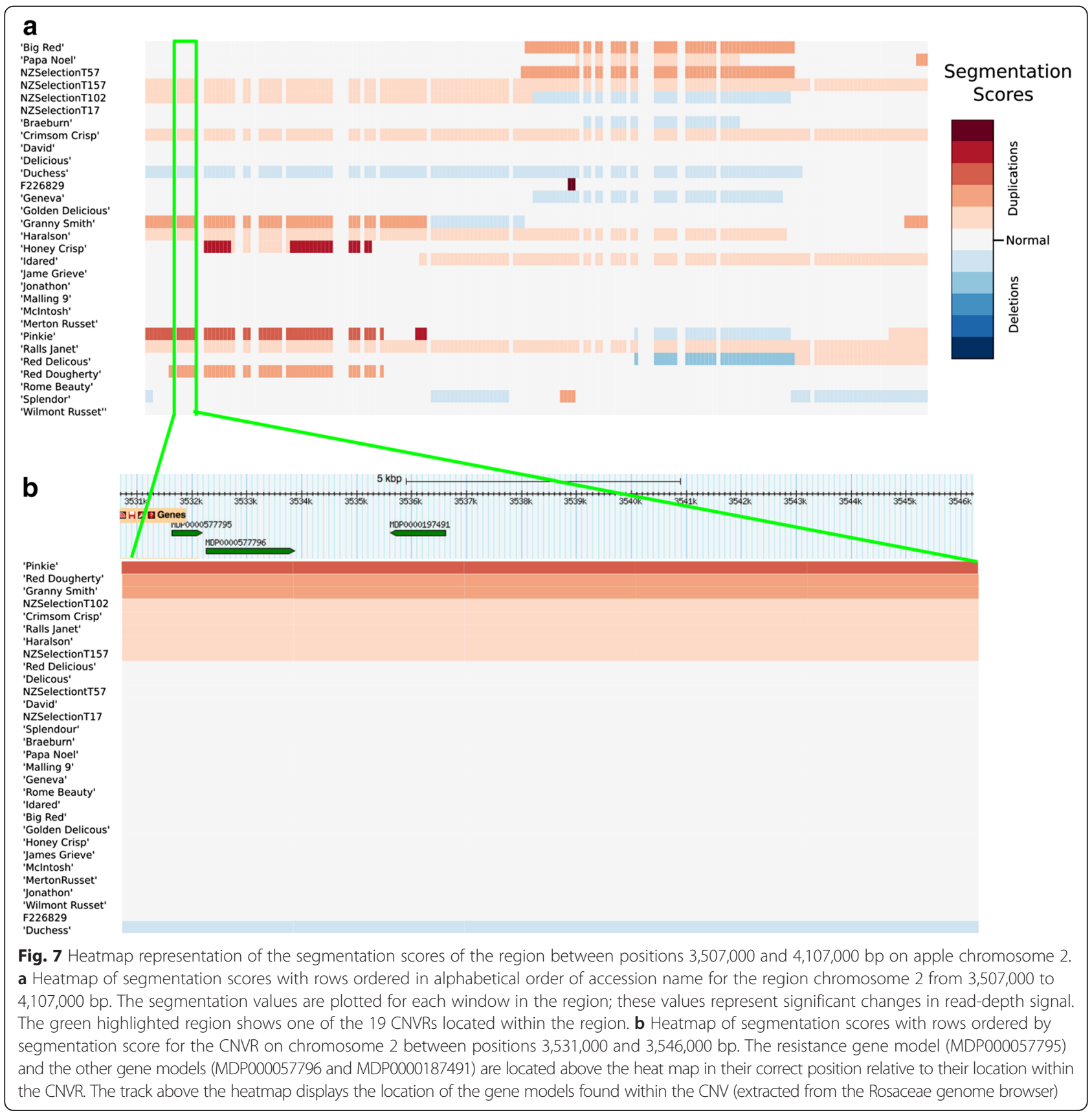




\section{In the apple genome CNVRs are enriched in resistance genes}

In total, 845 gene models overlapped the CNVRs detected. Initial functional enrichment analysis suggests that these gene models are involved in ion transport, signal transduction, and the defense response. This result is concordant with other studies in a wide range of species, such as humans [4], barley [3], and soybean [10], which have noted that immunity-related genes are frequently found within $\mathrm{CNV}$ regions. Fast-mutating CNVs have been recognized as an evolutionary driving force for organisms to adapt to changes in environmental conditions and the introduction of new pests and diseases [58]. This phenomenon is particularly important for long-lived tree species, which have a considerably lengthier generation time than their pathogens and which cannot migrate large distances to adapt to new conditions. In light of this, we propose that $\mathrm{CNV}$ is an extremely important adaptive genetic mechanism in tree species, even more so than for other organisms.

\section{Co-location between CNVRs and trait loci in apple}

Numerous studies have focused on identifying genomic regions associated with horticultural traits in apple, using genetic linkage mapping and QTL analysis [see Troggio et al. for a review [59]. Three regions of the apple genome that contain putative CNVRs are of particular interest, as they co-locate with loci controlling traits related to biotic and abiotic responses in apple. Firstly, a cluster of 19 CNVRs between 3,507,000 bp and 4,107,000 bp on chromosome 2 contains multiple $\mathrm{R}$ genes models (Figs. 6 \& 7) in a region where major loci conferring resistance to apple scab have been previously mapped. The $R v i 4 / V h 4$ and $R v i 15 / V r 2$ loci were originally mapped to apple linkage group $2[60,61]$ and the recent development of genetic markers closely linked to these loci enabled to estimate their physical location on the reference apple genome of 'Golden Delicious' [62], close to the detected cluster of 19 CNVRs. Secondly, a CNVR located at positions 3,506,106 and $3,509,841$ bp on chromosome 11 co-locates with Pl2, a major gene conferring resistance to powdery mildew. Pl2 was originally mapped using expressed sequence tags-based markers [62] and the newly developed markers for $P l 2$ [51] enabled placement of this locus on the apple genome sequence in the same position as the CNVR. Finally, three CNVRs located between positions 1,404,000 and 1,671,000 bp on chromosome 9 co-locate with a QTL for budbreak date [50]. Future work needs to include validation of these CNVRs, to demonstrate linkages between the CNVRs on chromosomes 2,11 , and 9 for scab resistance, powdery mildew resistance and budbreak date, respectively. This validation can be carried out by re-sequencing, with higher depth of coverage, cultivars carrying the scab resistance, powdery mildew, and early budbreak date alleles, and comparing the resulting integer copy-number genotypes to those of cultivars not carrying the resistance. Using the higher coverage data, the exact breakpoint of an individual CNV can be determined, and in combination with the integer copy-number genotypes, will enable the design of genetic markers that will accurately quantify the copy number at these loci. In addition to extensive genetic validation, functional validation of CNVs should also be performed. This could be achieved by assessing the mRNA/protein expression of the candidate gene(s) (in an appropriate tissue) from accessions that have different copy-numbers. A significant positive correlation would suggest a functional link, where raised copy-number increased the expression of the candidate gene(s). We hypothesize that some of these CNVs may be causative for these loci and that these functional $\mathrm{CNV}$ markers will be more powerful than nearby SNP markers for application in marker-assisted breeding.

\section{Conclusion}

We identified $876 \mathrm{CNVRs}$ with an average size of $16.4 \mathrm{~kb}$, comprising $3.5 \%$ of the apple genome. These results represent the first catalogue and investigation of a previously unexplored form of genetic variation in a tree species. The CNVRs identified in this study are enriched in resistance $(R)$ gene models and overlap with major gene loci of agricultural significance. Further investigation of apple CNV using higher coverage NGS data will enable integer-level copy-number assignment and break-point identification. This will facilitate the discovery of the causative $\mathrm{CNV}$ and improve the design of molecular markers that segregate with the trait. Ultimately, we believe that the focused investigation of $\mathrm{CNV}$ in the apple genome will lead to the genetic improvement of apple cultivars and a deeper understanding of the role CNV plays within the apple genome and other long-lived tree species.

\section{Additional files}

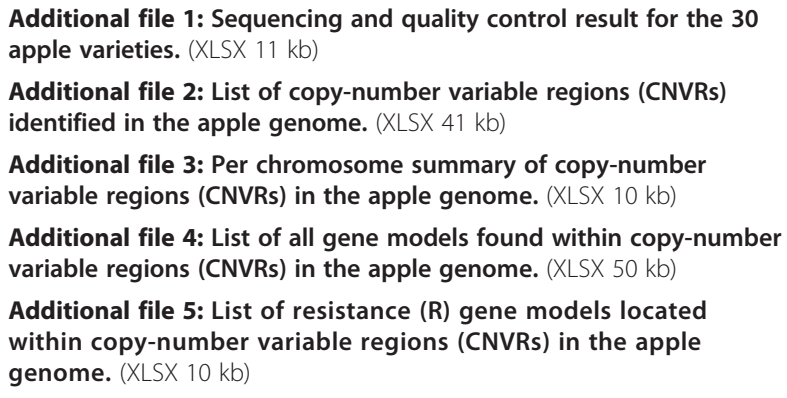

Additional file 5: List of resistance ( $R$ ) gene models located within copy-number variable regions (CNVRs) in the apple genome. (XLSX $10 \mathrm{~kb}$ )

Competing interests

The authors declare that they have no competing interests. 


\section{Authors' contributions}

JB performed the bioinformatics analysis and drafted the manuscript. DC contributed the sequence data. DC, TRM and MBA designed the study, coordinated the project and were involved in drafting the manuscript. All authors read and approved the final manuscript.

\section{Acknowledgements}

Computational resources from the New Zealand eScience Infrastructure (NeSI) were used for some of the analyses presented here (www.nesi.org.nz). We thank Hoang tan Nguyen (CNVrd2 developer), Tanya Flynn, Anna Gosling, and Murray Cadzow for their useful discussions throughout the course of this project, and Sue Gardiner and Susan Thompson from PFR for useful comments on the manuscript. Funding for this research was obtained from University of Otago, Plant \& Food Research's Core funding and the USDA RosBREED project.

\section{Author details}

'Department of Biochemistry, University of Otago, Dunedin, New Zealand. ${ }^{2}$ The Virtual Institute of Statistical Genetics (VISG), Rotorua, New Zealand. ${ }^{3}$ The New Zealand Institute for Plant \& Food Research Ltd, Palmerston North, New Zealand.

\section{Received: 13 July 2015 Accepted: 15 October 2015}

\section{Published online: 23 October 2015}

\section{References}

1. Girirajan S, Campbell CD, Eichler EE. Human Copy Number Variation and Complex Genetic Disease. Annu Rev Genet. 2011;45:203-26.

2. Nicholas TJ, Cheng Z, Ventura M, Mealey K, Eichler EE, Akey JM. The genomic architecture of segmental duplications and associated copy number variants in dogs. Genome Res. 2009;19:491-9.

3. Muñoz-Amatriaín M, Eichten SR, Wicker T, Richmond TA, Mascher M, Steuernagel B, et al. Distribution, functional impact, and origin mechanisms of copy number variation in the barley genome. Genome Biol. 2013;14:R58

4. Conrad DF, Pinto D, Redon R, Feuk L, Gokcumen O, Zhang Y, et al. Origins and functional impact of copy number variation in the human genome. Nature. 2009:464:704-12.

5. Kurotaki N, Shen JJ, Touyama M, Kondoh T, Visser R, Ozaki T, et al. Phenotypic consequences of genetic variation at hemizygous alleles: Sotos syndrome is a contiguous gene syndrome incorporating coagulation factor twelve (FXII) deficiency. Genet Med. 2005;7:479-83.

6. Drögemüller C, Distl O, Leeb T. Partial deletion of the bovine ED1 gene causes anhidrotic ectodermal dysplasia in cattle. Genome Res. 2001;11:1699-705.

7. Pielberg G, Olsson C, Syvänen A-C, Andersson L. Unexpectedly high allelic diversity at the KIT locus causing dominant white color in the domestic pig. Genetics. 2002;160:305-11.

8. Pielberg G, Day AE, Plastow GS, Andersson L. A sensitive method for detecting variation in copy numbers of duplicated genes. Genome Res. 2003;13:2171-7.

9. Sharp AJ, Locke DP, McGrath SD, Cheng Z, Bailey JA, Vallente RU, et al. Segmental duplications and copy-number variation in the human genome. Am J Hum Genet. 2005;77:78-88.

10. Zhou Z, Jiang Y, Wang Z, Gou Z, Lyu J, Li W, et al. Resequencing 302 wild and cultivated accessions identifies genes related to domestication and improvement in soybean. Nat Biotechnol. 2015:33:408.

11. Springer NM, Ying K, Fu Y, Ji T, Yeh C-T, Jia Y, et al. Maize inbreds exhibit high levels of copy number variation (CNV) and presence/absence variation (PAV) in genome content. PLoS Genet. 2009:5:e1000734.

12. Díaz A, Zikhali M, Turner AS, Isaac P, Laurie DA. Copy number variation affecting the Photoperiod-B1 and Vernalization-A1 genes is associated with altered flowering time in wheat (Triticum aestivum). PLoS One. 2012;7:e33234.

13. Pearce S, Saville R, Vaughan SP, Chandler PM, Wilhelm EP, Sparks CA, et al. Molecular characterization of Rht-1 dwarfing genes in hexaploid wheat. Plant Physiol. 2011;157:1820-31.

14. Cook DE, Lee TG, Guo X, Melito S, Wang K, Bayless AM, et al. Copy number variation of multiple genes at Rhg1 mediates nematode resistance in soybean. Science (80-). 2012:338:1206-9.

15. Sutton T, Baumann U, Hayes J, Collins NC, Shi B-J, Schnurbusch T, et al. Borontoxicity tolerance in barley arising from efflux transporter amplification. Science (80-). 2007;318:1446-9.
16. Velasco R, Zharkikh A, Affourtit J, Dhingra A, Cestaro A, Kalyanaraman A, et al. The genome of the domesticated apple (Malus x domestica Borkh.). Nat Genet. 2010;42:833-9.

17. Kumar S, Chagné D, Bink MCAM, Volz RK, Whitworth C, Carlisle C. Genomic selection for fruit quality traits in apple (Malus $\times$ domestica Borkh.). PLoS One. 2012;7:1-10.

18. Bianco L, Cestaro A, Sargent DJ, Banchi E, Derdak S, Di Guardo M, et al. Development and validation of a 20K Single Nucleotide Polymorphism (SNP) whole genome genotyping array for apple (Malus $x$ domestica Borkh). PLoS One. 2014;9:e110377.

19. Chagne D, Crowhurst RN, Troggio M, Davey MW, Gilmore B, Lawley C, et al. Genome-wide SNP detection, validation, and development of an 8K SNP array for apple. PLoS One. 2012;7:e31745.

20. Cornille A, Gladieux P, Smulders MJM, Roldán-Ruiz I, Laurens F, Le Cam B, et al. New insight into the history of domesticated apple: secondary contribution of the European wild apple to the genome of cultivated varieties. PLoS Genet. 2012:8:e1002703.

21. Redon R, Ishikawa S, Fitch KR, Feuk L, Perry GH, Andrews TD, et al. Global variation in copy number in the human genome. Nature. 2006:444:444-54

22. Zhao M, Wang Q, Wang Q, Jia P, Zhao Z. Computational tools for copy number variation (CNV) detection using next-generation sequencing data: features and perspectives. BMC Bioinformatics. 2013;14 Suppl 11:S1.

23. $\tan$ Nguyen $H$, Merriman TR, Black MA. CNVrd, a read-depth algorithm for assigning copy-number at the FCGR locus: population-specific tagging of copy number variation at FCGR3B. PLoS One. 2013;8:e63219.

24. Li H, Durbin R. Fast and accurate short read alignment with Burrows-Wheeler transform. Bioinformatics. 2009:25:1754-60.

25. Li H. Aligning sequence reads, clone sequences and assembly contigs with BWA-MEM. arXiv Prepr arXiv13033997 2013. http:// www.genomebiology.com/2014/15/6/R84\#B26

26. Kielbasa SM, Wan R, Sato K, Horton P, Frith MC. Adaptive seeds tame genomic sequence comparison. Genome Res. 2011:21:487-93.

27. Apple mitochondria reference. http://www.rosaceae.org/sites/default/files/ apple_genome/Malus_x_domestica.v1.0.mitochondria.fa.gz. Accessed Oct 2015 .

28. Apple chloroplast reference. http://www.rosaceae.org/sites/default/files/ apple_genome/Malus_x_domestica.v1.0.chloroplast.fa.gz. Accessed Oct 2015.

29. Apple repeat sequences. ftp://ftp.bioinfo.wsu.edu/species/Malus_x_domestica/ Malus_x_domestica-genome.v1.0-primary_haplotype/repeats/

Malus_x_domestica.v1.0-primary.repeats.gff3.gz. Accessed Oct 2015.

30. Quinlan AR, Hall IM. BEDTools: a flexible suite of utilities for comparing genomic features. Bioinformatics. 2010;26:841-2.

31. Picard software. http://broadinstitute.github.io/picard/. Accessed Oct 2015.

32. Nguyen HT, Merriman TR, Black MA. The CNVrd2 package: measurement of copy number at complex loci using high-throughput sequencing data. Front Genet. 2014;5.

33. Dohm JC, Lottaz C, Borodina T, Himmelbauer H. Substantial biases in ultra-short read data sets from high-throughput DNA sequencing. Nucleic Acids Res. 2008;36:e105-e105.

34. R Core Team. R: A language and environment for statistical computing. 2014.

35. Wickham H. ggplot2: elegant graphics for data analysis. New York: Springer; 2009.

36. Warnes GR, Bolker B, Bonebakker L, Gentleman R, Huber W, Liaw A, et al. Gplots: Various R programming tools for plotting data. R Packag version 2009; 2. http:// cran.rproject.org/web/packages/gplots/index.html (2011)http://cran.rproject.org/ web/packages/gplots/index.html(2011)

37. Venkatraman ES, Olshen AB. A faster circular binary segmentation algorithm for the analysis of array CGH data. Bioinformatics. 2007;23: 657-663.

38. Gentleman RC, Carey VJ, Bates DM, Bolstad B, Dettling M, Dudoit S, et al. Bioconductor: open software development for computational biology and bioinformatics. Genome Biol. 2004;5:R80

39. Krzywinski M, Schein J, Birol I, Connors J, Gascoyne R, Horsman D, et al. Circos: an information aesthetic for comparative genomics. Genome Res. 2009:19:1639-45.

40. Klambauer G, Schwarzbauer K, Mayr A, Clevert DA, Mitterecker A, Bodenhofer $U$, et al. cn.MOPS: mixture of Poissons for discovering copy number variations in next-generation sequencing data with a low false discovery rate. Nucleic Acids Res. 2012;40:9.

41. Benjamini $Y$, Hochberg $Y$. Controlling the false discovery rate: a practical and powerful approach to multiple testing. J R Stat Soc Ser B 1995;289-300. 
42. Wang K, Li M, Hadley D, Liu R, Glessner J, Grant SFA, et al. PennCNV: an integrated hidden Markov model designed for high-resolution copy number variation detection in whole-genome SNP genotyping data Genome Res. 2007;17:1665-74.

43. Fisher RA. On the interpretation of $\left\{X^{\wedge} 2\right\}$ from contingency tables, and the calculation of P. J R Stat Soc. 1922;85:87-94.

44. Apple genes. http://www.rosaceae.org/sites/default/files/apple_genome/ Malus x domestica.v1.0-primary.transcripts.gff3.gz. Accessed Oct 2015.

45. Ashburner M, Ball CA, Blake JA, Botstein D, Butler H, Cherry JM, et al. Gene Ontology: tool for the unification of biology. Nat Genet. 2000;25:25-9.

46. Altschul SF, Gish W, Miller W, Myers EW, Lipman DJ. Basic local alignment search tool. J Mol Biol. 1990;215:403-10.

47. Jurka J, Kapitonov W, Pavlicek A, Klonowski P, Kohany O, Walichiewicz J. Repbase Update, a database of eukaryotic repetitive elements. Cytogenet Genome Res. 2005;110:462-7.

48. Eitas TK, Dangl JL. NB-LRR proteins: pairs, pieces, perception, partners, and pathways. Curr Opin Plant Biol. 2010;13:472-7.

49. Dangl JL, Jones JDG. Plant pathogens and integrated defence responses to infection. Nature. 2001:411:826-33.

50. Celton J-M, Martinez S, Jammes M-J, Bechti A, Salvi S, Legave J-M, et al. Deciphering the genetic determinism of bud phenology in apple progenies: a new insight into chilling and heat requirement effects on flowering dates and positional candidate genes. New Phytol. 2011;192:378-92.

51. Jänsch $M$, Broggini GAL, Weger J, Bus VGM, Gardiner SE, Bassett $H$, et al. Identification of SNPs linked to eight apple disease resistance loci. Mol Breed. 2015:35:1-21.

52. Zmieńko A, Samelak A, Kozłowski P, Figlerowicz M. Copy number polymorphism in plant genomes. Theor Appl Genet. 2014;127:1-18.

53. Malus $x$ domestica Whole Genome v1.0p Assembly. http:// www.rosaceae.org/sites/default/files/apple_genome/ Malus_x_domestica.v1.0-primary.pseudo.fa.gz. Accessed Oct 2015.

54. Paudel $Y$, Madsen $O$, Megens H-J, Frantz LAF, Bosse M, Bastiaansen JWM, et al. Evolutionary dynamics of copy number variation in pig genomes in the context of adaptation and domestication. BMC Genomics. 2013;14:449.

55. Bickhart DM, Hou Y, Schroeder SG, Alkan C, Cardone MF, Matukumalli LK, et al Copy number variation of individual cattle genomes using next-generation sequencing. Genome Res. 2012;22:778-90.

56. Jiao Y, Zhao H, Ren L, Song W, Zeng B, Guo J, et al. Genome-wide genetic changes during modern breeding of maize. Nat Genet. 2012;44:812-5.

57. Ching ADA, Caldwell KS, Jung M, Dolan M, Smith OSH, Tingey S, et al. SNP frequency, haplotype structure and linkage disequilibrium in elite maize inbred lines. BMC Genet. 2002;3:19.

58. Kondrashov FA. Gene duplication as a mechanism of genomic adaptation to a changing environment. Proc R Soc B Biol Sci. 2012;279(September):5048-57.

59. Troggio M, Gleave A, Salvi S, Chagné D, Cestaro A, Kumar S, et al. Apple, from genome to breeding. Tree Genet Genomes. 2012;8:509-29.

60. Bus VGM, Rikkerink EHA, Van De Weg WE, Rusholme RL, Gardiner SE, Bassett HCM, et al. The Vh2 and Vh4 scab resistance genes in two differential hosts derived from Russian apple R12740-7A map to the same linkage group of apple. Mol Breed. 2005;15:103-16

61. Patocchi A, Bigler B, Koller B, Kellerhals M, Gessler C. Vr2: a new apple scab resistance gene. Theor Appl Genet. 2004;109:1087-92.

62. Gardiner S, Murdoch J, Meech S, Rusholme R, Bassett H, Cook M, et al. Candidate resistance genes from an EST database prove a rich source of markers for major genes conferring resistance to important apple pests and diseases. Acta Hortic. 2003;622:141-151. http://www.actahort.org/books/622/ 622_12.htm

\section{Submit your next manuscript to BioMed Central and take full advantage of:}

- Convenient online submission

- Thorough peer review

- No space constraints or color figure charges

- Immediate publication on acceptance

- Inclusion in PubMed, CAS, Scopus and Google Scholar

- Research which is freely available for redistribution

Submit your manuscript at www.biomedcentral.com/submit 